\title{
Characterization of the $\mathrm{c} .190 \mathrm{~T}>\mathrm{C}$ missense mutation in BRCA1 codon 64 (Cys64Arg)
}

\author{
P. WILLEMS ${ }^{1}$, V. MAGRI ${ }^{2}$, M. CRETNIK ${ }^{3}$, M. FASANO ${ }^{4}$, A. JAKUBOWSKA ${ }^{5}$, \\ S. LEVANAT ${ }^{3}$, J.LUBINSKI ${ }^{5}$, E. MARRAS ${ }^{4}$, V. MUSANI $^{3}$, H. THIERENS ${ }^{1}$, \\ V. VANDERSICKEL ${ }^{1}$, G. PERLETTI ${ }^{4 *}$ and A. VRAL ${ }^{1 *}$ \\ ${ }^{1}$ Department of Basic Medical Sciences, Ghent University, Ghent, Belgium; ${ }^{2}$ Istituti Clinici \\ di Perfezionamento, Milan, Italy; ${ }^{3}$ Division of Molecular Medicine, Rudjer Boskovic Institute, Zagreb, \\ Croatia; ${ }^{4}$ Department of Structural and Functional Biology, Università degli Studi dell'Insubria, Varese, \\ Italy; ${ }^{5}$ Department of Genetics and Pathology, Pomeranian Medical University, Szczecin, Poland
}

Received October 30, 2008; Accepted December 2, 2008

DOI: 10.3892/ijo_00000226

\begin{abstract}
In the Milan area (Northern Italy), we identified a family characterized by a high prevalence of ovarian and breast cancer cases ( 5 out of 6 subjects, over 3 generations), and a predominant prevalence of ovarian lesions (4 out of 5 patients). Analysis of $B R C A 1$ and $B R C A 2$ genes allowed the identification of the missense c.190T $>C$ mutation in codon 64 (Cys64Arg) of BRCA1. The aims of the present investigation were to characterize the functional implications of the c.190T $>$ C mutation at the molecular level, and to search whether additional polymorphisms might be linked to the peculiar phenotypic features observed in the Italian pedigree. Molecular modelling studies suggested that substitution of the cysteine 64 with an arginine likely disrupts the architecture of the BRCA1 RING finger domain, responsible for the interaction with BARD1, essential for the tumor-suppressor activity of the BRCA1-BARD1 complex. By splicing site information analysis, exonic splicing enhancer site characterization, and analysis of transcript fragment length and sequence, we showed that the c.190T $>C$ mutation was able to modulate the splicing of exon 5 in a fashion opposite to the c.190T $>\mathrm{G}$ transversion, responsible for the functionally-related
\end{abstract}

Correspondence to: Dr Gianpaolo Perletti, Università degli Studi dell'Insubria, Dipartimento di Biologia Strutturale e Funzionale, Via A. Da Giussano, 12, 21052 Busto A., Varese, Italy

E-mail: gianpaolo.perletti@uninsubria.it

${ }^{*}$ Contributed equally

Abbreviations: BIC, breast cancer information core; EBV, Epstein-Barr virus; HBOC, hereditary breast and ovarian cancer; IC, information content; $\mathrm{MN}$, micronucleus; SNP, single nucleotide polymorphism

Key words: breast cancer, BRCA1, BRCA2, missense mutation, RING finger, BARD1, splicing
Cys64Gly amino acid substitution. Genotyping of BRCAI and $B R C A 2$ in the Italian family revealed the presence of two significant polymorphisms: the cancer-associated c. $2612 \mathrm{C}>\mathrm{T}$ $\mathrm{SNP}$ in $B R C A 1$, and the c.-26G $>\mathrm{A} \mathrm{SNP}$ in the $B R C A 2$ gene, acting as an ovarian cancer risk modifier in carriers of deleterious BRCAl mutations. Analysis of these SNPs in a genotypically-unrelated Polish family, characterized by prevalent breast neoplasms in carriers of the c.190T $>C$ mutation, revealed a genetic profile consistent with the hypothetic role of both polymorphisms.

\section{Introduction}

Hereditary breast cancer accounts for $5-10 \%$ of all breast cancers worldwide. Prevalence of $B R C A 1$ and $B R C A 2$ mutations among high-risk cancer patients may vary by ethnicity, study inclusion criteria and mutation detection techniques. Since their early characterization, inherited BRCAl mutations were found to be responsible for $\sim 45 \%$ of inherited early-onset breast cancers, and for $>80 \%$ of inherited breast and ovarian cancers (1). In Europe, BRCAl mutation frequencies in breast cancer populations show considerable variability and range between $0.4 \%$ in Finland and $7 \%$ in Sweden (2). Meta-analytic mean cumulative cancer risks for mutation carriers at age 70 years are $57 \%$ for BRCAl and $49 \%$ for $B R C A 2$ mutation carriers; ovarian cancer risk is $40 \%$ for $B R C A 1$ and $18 \%$ for BRCA2 mutation carriers (3). In Italy, breast cancer penetrance, estimated in a series of 568 families, was found to be $27 \%$ at age 50 and $39 \%$ at age 70 in BRCAI mutation carriers, and $26 \%$ at age 50 and $44 \%$ at age 70 in BRCA2 mutation carriers (4). Ovarian cancer penetrances in Italy were estimated to be $14 \%$ at age 50 and $43 \%$ at age 70 in BRCA1 mutation carriers, and $3 \%$ at age 50 and $15 \%$ at age 70 in $B R C A 2$ mutation carriers (4).

$B R C A 1$ is a gene with a coding sequence spanning over 23 exons, including the large exon 11 , that holds more than half of the coding potential of the gene (5). The major BRCAI product comprises 1863 amino acids and can be subdivided into three major domains: a RING domain adjacent to the amino-terminus, tandem-repeated C-terminal BRCT domains, 
and a central domain, including the large region encoded by exon 11 .

The function of the poorly conserved central domain is not fully understood, although some evidence suggests interaction with RAD51 (6). The structure of BRCT motifs is similar to C-terminal domains found in a number of proteins involved in cell cycle checkpoints and DNA repair (reviewed in ref. 7). BRCT motifs were shown to activate gene transcription when linked to a DNA-binding domain (8).

The RING domain is probably the better-characterized structure of BRCA1. It encompasses amino acid residues 24-64 and contains a Cys3-Hys-Cys4 $\left(\mathrm{C}_{3} \mathrm{HC}_{4}\right)$ motif, which has been shown to allow interaction with proteins involved in cell-cycle regulation, such as cyclins and cyclin-dependent kinases (9), and with estrogen receptors (10). A major binding partner is the BRCA1-associated RING domain protein 1 (BARD1). Originally identified due to its interaction with the RING domain of BRCA1 (11), BARD1 is required for S phase progression, contact inhibition and normal nuclear division. BRCA1 acquires significant ubiquitin ligase activity when bound to BARD1 as a RING heterodimer, and catalyzes Lys6-linked polyubiquitin chains, which are recognized in vitro by the $26 \mathrm{~S}$ proteasome for deubiquitination as opposed to degradation (12).

Pathogenic alterations in BRCAl include mutations in the regulatory regions of the gene, splice site mutations, nonsense/ frameshift/missense mutations, and large chromosomal rearrangements, estimated to account for $\sim 19 \%$ of all BRCAI mutations in Italian patients (13).

In Northern Italy, we identified a family characterized by a high prevalence of neoplasia: of a total of 6 women in a 3generation maternal lineage, 4 were affected by ovarian cancer, and one by early-onset bilateral breast cancer associated with ovarian dysplasia and abnormalities. The only non-diseased subject showed breast dysplastic lesions. In two patients belonging to the youngest generation and treated with a modern approach, a good prognosis and longterm survival was observed. Although family history was collected at diagnosis or hospitalization, patients were not subjected to genetic analysis, nor were treated as members of a HBOC pedigree.

To identify the molecular determinants of the hereditary breast and ovary cancer (HBOC) syndrome in this family, we analyzed the BRCA1 and BRCA2 genotypes of one patient, and extended to the rest of the family and to a family from Poland without Italian ancestry some observations about the peculiar genotype/phenotype of this family. In addition, we performed a molecular study, both in vitro and in silico, to characterize at the functional level the gene and protein alterations associated with the c.190T $>$ C (Cys64Arg) mutation.

\section{Materials and methods}

Detection of BRCA1 and BRCA2 mutations and polymorphisms High-resolution melting analysis. DNA was extracted from peripheral blood leukocytes of Patient 1 (Fig. 1), and the entire coding sequence and exon-intron boundaries were amplified using the polymerase chain reaction (PCR). Thirty-six PCR products (size range: $150-437$ bp for $B R C A 1$ ) and 49 PCR products (179-500 bp for $B R C A 2)$ were amplified (14). PCR

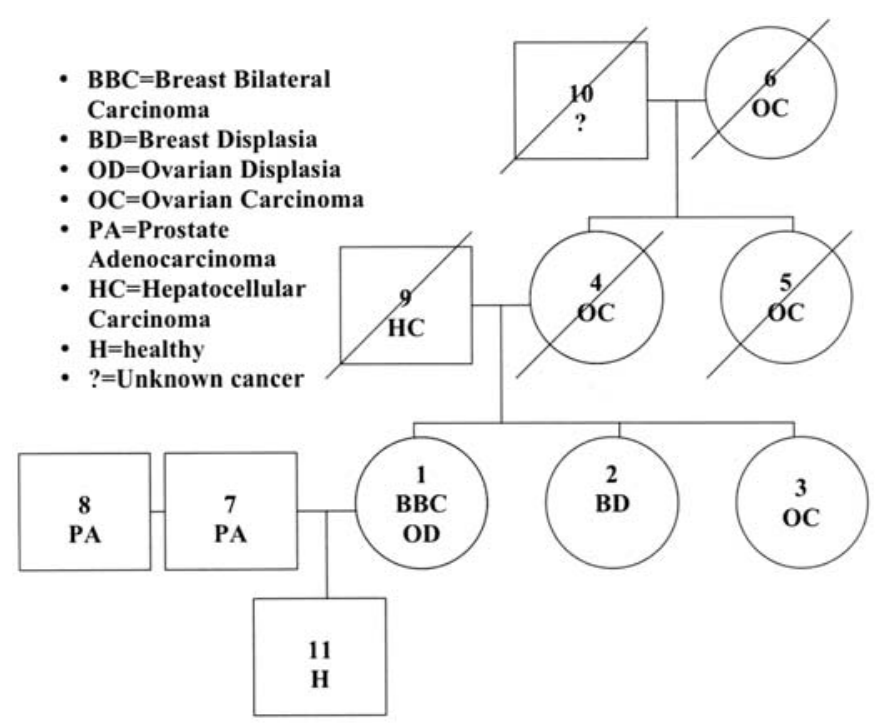

Figure 1. Schematic pedigree representing the Italian HBOC family described in this study. The abbreviation legend is embedded in the picture. The mother lineage of proband 11, comprising all breast and ovarian cancer patients is complete; some members of the father lineage of proband 11 are not reported for lack of information. However, all significant cancer patients from both lineages of up to three generations are reported.

was performed in $10 \mu 1$ reaction mixture containing template DNA, dNTPs (Roche, Mannheim, Germany), FastStart Taq DNA Polymerase (Roche), fluorescent dye LCGreen Plus (Idaho Technology) and forward and reverse primers for each gene segment, in Roche LightCycler capillaries and amplified in an adapted RapidCycler2 instrument (Idaho Technology). PCRs were performed using appropriate sets of primers as described (14), with slight modifications.

PCR conditions were optimized to Tm between 49 and $68^{\circ} \mathrm{C}$ for each segment. After 40 cycles of amplification, PCR products underwent an additional cycle of $1 \mathrm{~min}$ at $98^{\circ} \mathrm{C}$, followed by $5 \mathrm{~min}$ at $40^{\circ} \mathrm{C}$ to promote heteroduplex formation. Reaction products were then transferred to a high-resolution melter instrument (HR-1, Idaho Technology) for highresolution melting and curve analysis. Samples were melted at $0.2^{\circ} \mathrm{C} / \mathrm{sec}$ ramp rate. Melting profiles were analyzed with the HR-1 software using fluorescence normalization, temperature shift and conversion to difference and derivative plots. Fragments with melting patterns different from the wild-type were sequenced to determine the exact sequence alterations.

Nucleotide numbering was according to BIC database, where position 1 represents the first nucleotide of exon 1 . Reference sequences used were NG_005905.1 for BRCA1, and NW_001838072.1 for BRCA2.

Before sequencing, PCR products were purified with ExoSAP-IT (USB, Cleveland, USA), and then sequenced in both directions using the Big Dye terminator 1.1 cycle sequencing kit (Applied Biosystems, Foster City, USA). Sequencing analysis was performed on an automatic sequencer ABI PRISM 310 genetic analyzer (Applied Biosystems).

RNA-based sequencing. In the Polish pedigree, detection of the c.190T $>$ C mutation in BRCAl was performed by sequencing RT-PCR products, amplified from RNA purified from peripheral blood lymphocytes of patients and relatives, as previously described (15). 
Table I. PCR primers for genotyping the c.190T>C (Cys64Arg) mutation in BRCA1, and SNaPshot primers for genotyping the c.2612C $>$ T (Pro871Leu) SNP in BRCA1 and the c.-26G>A SNP in BRCA2.

\begin{tabular}{llc}
\hline Mutation/polymorphism & \multicolumn{1}{c}{ PCR primers } & SNaPshot primer \\
\hline BRCA1: c.190T>C (Cys64Arg) & F - GCCTTTTGAGTATTCTTTCTAC & // \\
BRCA1: c.2612C>T (Pro871Leu) & F - GGAAGGCAAAAACAGAAC & GTTTCAAAGCGCCAGTCATTTGCTC \\
& R - CCAACCACAGGAAAGCCT & \\
BRCA2: c.-26G>A & F - TGTTACCTTCCAGGAGATG & CATTTTTACCTACGATATTCCTCCAATG \\
& R - AGCAACACTGTGACGTACTG & \\
\hline
\end{tabular}

F, forward; R, reverse.

PCR-RFLP and SNapShot analysis. To detect the c.190T $>C$ (Cys64Arg) and c.2612C $>$ T (Pro871Leu) variants in $B R C A 1$, and the c.-26G $>\mathrm{A}$ noncoding variant in $B R C A 2$ in Italian and Polish patients, i) PCR combined with restriction fragment length polymorphism (RFLP) analysis, or ii) SNaPshot techniques were used.

First, PCR products were amplified using 100-200 ng DNA in a $25 \mu 1$ reaction containing $0.5 \mathrm{mM}$ dNTPs (Amersham Bioscience), 1X PCR buffer (Invitrogen), $1.5 \mathrm{mM} \mathrm{MgCl}_{2}$ (Invitrogen), $1 \mathrm{mM}$ forward and reverse primer (Invitrogen) and 0.6 units Platinum Taq polymerase (Invitrogen). The sequence of the primers used for each mutation or SNP can be found in Table I.

The DNA fragment containing the Cys64Arg mutation in BRCA1 was amplified using a 35-cycle PCR program consisting of an initial denaturation step at $95^{\circ} \mathrm{C}$ for $5 \mathrm{~min}$, 35 cycles of $3 \mathrm{~min}$ with a denaturation step at $95^{\circ} \mathrm{C}(1 \mathrm{~min})$, an annealing step at $58^{\circ} \mathrm{C}(1 \mathrm{~min})$, and an elongation step at $72^{\circ} \mathrm{C}(1 \mathrm{~min})$, followed by a final extension step of $10 \mathrm{~min}$ at $72^{\circ} \mathrm{C}$. After DNA amplification, PCR products were digested $\left(3 \mathrm{~h}\right.$ at $37^{\circ} \mathrm{C}$ ) using the specific restriction endonuclease SnaB1 (New England Biolabs) which recognized and cut the variant sequence. Digested products were analysed by gel electrophoresis on a $2 \%$ agarose gel and visualised under ultraviolet light after ethidium bromide staining.

The PCR program for the c.2612C $>$ T SNP (Pro871Leu) in BRCAl and the c.-26G>A SNP in BRCA2 consisted of an initial denaturation step of $94^{\circ} \mathrm{C}, 12$ cycles of denaturation at $94^{\circ} \mathrm{C}$ for $20 \mathrm{sec}$, annealing at $58^{\circ} \mathrm{C}$ and elongation at $72^{\circ} \mathrm{C}$ for $1 \mathrm{~min}$. At each cycle, the annealing temperature decreased by $1{ }^{\circ} \mathrm{C}$. This was followed by 24 cycles of 40 seconds at $94^{\circ} \mathrm{C}$, $40 \mathrm{sec}$ at $46^{\circ} \mathrm{C}$ and $30 \mathrm{sec}$ at $72^{\circ} \mathrm{C}$ and a final extension step at $72^{\circ} \mathrm{C}$ (10 min). Further analyses used the SNapShot multiplex system (Applied Biosystems); the SNapShot primers are listed in Table I. Analyses of $2 \mu 1$ of the SNaPshot product with $10 \mu \mathrm{l}$ Amresco capillary electrophoresis buffer (Lucron Bioproducts) and $0.3 \mu 1$ LIZ-120 size standard (Applied Biosystems) was performed on the capillary system of an ABI Prism 3730 genetic analyser (Applied Biosystems). Results were visualised on the Peak scanner program version 1.0 (Applied Biosystems).

In silico structural and functional analysis of the Cys64Gly mutation. The three-dimensional NMR structure of the
BRCA1-BARD1 RING finger heterodimer [PDB ID 1JM7; (16)] was downloaded from the Protein data bank (http://www. rcsb.org). Structural models were drawn with the SwissPdbViewer (17).

Analysis of splice sites was performed using the Information analysis tools at Schneider's lab, Molecular Information Theory Group, Center for Cancer Research Nanobiology Program, National Cancer Institute, Frederick, MD (http://www.lecb.ncifcrf.gov/ toms/delila.html). Exonic splicing enhancer analysis $(18,19)$ was performed using the ESEfinder 2.0 program, Cold Spring Harbor Laboratories (http://rulai.cshl.edu/cgi-bin/tools/ESE3/esefinder.cgi? process=home).

Immortalization of peripheral blood lymphocytes from patients and healthy individuals. EBV-immortalized cell lines from healthy individuals, homozygous (T/T) at the 109 locus, were obtained from the cell bank of Professor A. Vral, (University of Gent, Belgium). The EBV cell line BC1 was generated from peripheral blood lymphocytes of the Italian patient 1 (Fig. 1), heterozygous for the c.190T >C mutation in BRCA1. Immortalization was achieved by exposure of lymphocytes to the supernatants of the B95-8 EBV producer cell line, according to a standard protocol (20). EBV cell lines were used to amplify transcript sequences of the BRCAl gene by RT-PCR, and for radiosensitivity testing with the cytokinesisblock micronucleus (MN) assay, as previously described (20). The EBV cell lines were maintained in RPMI-1640 medium supplemented with $10 \%$ fetal bovine serum, $2 \mathrm{mM}$ L-glutamine, $100 \mathrm{U} / \mathrm{ml}$ penicillin, $0.1 \mathrm{mg} / \mathrm{ml}$ streptomycin with $10 \% \mathrm{FBS}$ and cultured at $37^{\circ} \mathrm{C}, 5 \% \mathrm{CO}_{2}$, in a humidified incubator.

Analysis of BRCA1 transcripts by RT-PCR. Total RNA was isolated from $10^{7} \mathrm{EBV}$-immortalized lymphoblastoid cells using the SV Total RNA isolation system (Promega Italy), according to manufacturer directions. A 2-3 $\mu \mathrm{g}$ aliquot of total RNA was used to generate first-strand cDNA and to amplify transcript fragments with a RobusT II RT-PCR kit (Finnzymes, Italy) at the following conditions: $48^{\circ} \mathrm{C}$ for $30 \mathrm{~min}$, followed by $94^{\circ} \mathrm{C}$ for 2 min for first-strand cDNA synthesis, followed by 35 amplification cycles $\left(94^{\circ} \mathrm{C}, 30 \mathrm{sec} ; 60^{\circ} \mathrm{C}\right.$, $60 \mathrm{sec} ; 72^{\circ} \mathrm{C}, 1 \mathrm{~min}$ ) and a final extension step at $72^{\circ} \mathrm{C}$ for $7 \mathrm{~min}$. To detect normal and/or alternative transcript fragments 

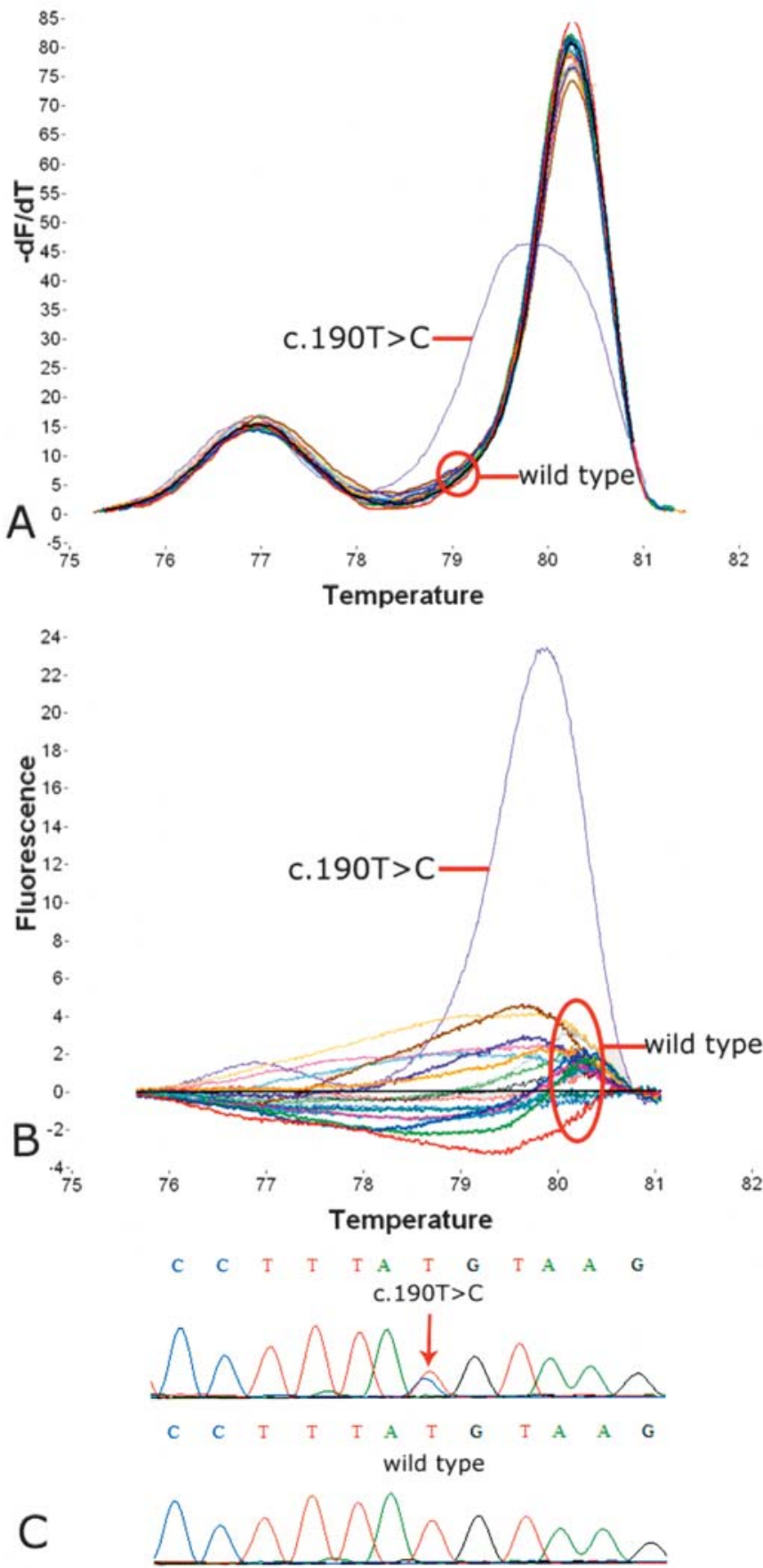

Figure 2. (A) High-resolution melting analysis of exon 5 of BRCA1, shown as a derivative plot $(-\mathrm{dF} / \mathrm{dT}$ vs. temperature). Mutation c.190T $>\mathrm{C}$ is clearly distinct from wild-type controls. (B) High-resolution melting analysis of exon 5 of $B R C A 1$, shown as difference plot (fluorescence vs. temperature), compared to a known wild-type sequence. (C) Sequences of exon 5 of BRCA1: the sample harboring c.190T $>\mathrm{C}$ is compared to a wild-type control. The mutation is marked with an arrow.

of BRCA1, exon 5, we designed a forward primer (5'-ATT TATCTGCTCTTCGCGTTG-3'), annealing with a specific sequence on exon 5, and a reverse primer (5'-CACTGAG ACTGGTTTCCTGC-3'), annealing on exon 6. In normal conditions a fragment of $456 \mathrm{bp}$ would be amplified by RTPCR. In case of alternative splicing, as described in cells harboring the c.190T $>$ C mutation (21), a 434-bp fragment would be detected. For transcript fragment length analysis, RT-PCR products were run on $2.5 \%$ agarose gels; fragments

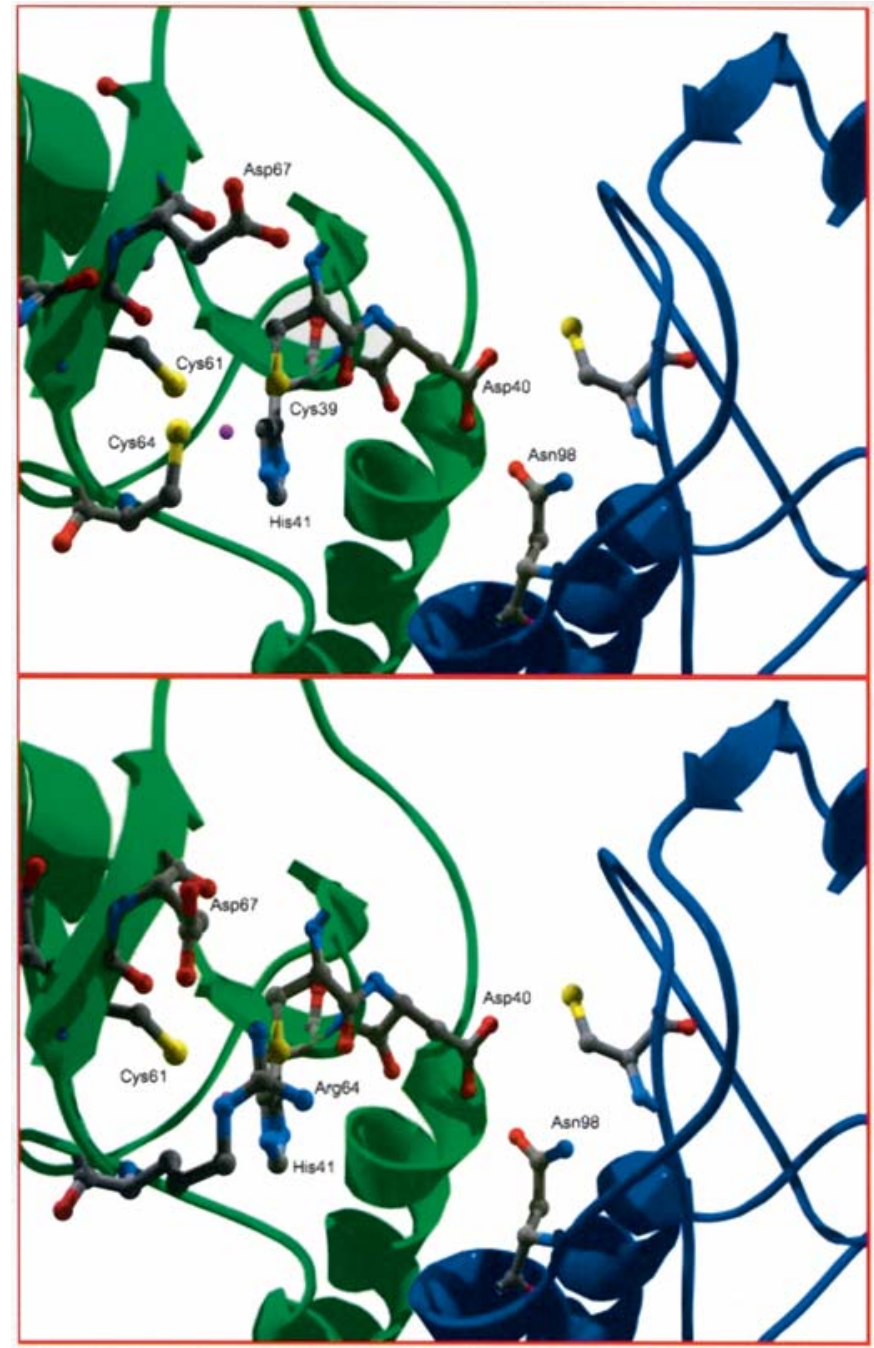

Figure 3. Ribbon representation of the $\mathrm{C}_{3} \mathrm{H}$ zinc coordination site in the BRCA1 N-terminal RING finger domain and of the BRCA1-BARD1 interaction interface (PDB ID: 1JM7). BRCA1 and BARD1 are shown in green and blue, respectively. Sidechains of selected amino acids are rendered in ball-and-stick representation. The wild-type protein is shown in the top panel. The $\mathrm{C}_{3} \mathrm{H}$ site stabilizes the BRCA1-BARD1 interaction, with a hydrogen bond between Asp40 of BRCA1 and Asn98 of BARD1. The Zinc atom coordinated by the $\mathrm{C}_{3} \mathrm{H}$ structure, including Cysteine 64 and Cysteine 61 , is represented as a small sphere in color magenta. The bottom panel shows the Cys64Arg mutant. Arginine 64 establishes a salt bridge with Aspartic acid 67, with the consequent destabilization of the Zinc coordination cage.

were analyzed by restriction digestion (presence of $\mathrm{T}>\mathrm{C}$ mutation was screened with $\mathrm{SnaBI}$ ) and automated sequencing.

\section{Results and Discussion}

Clinical and genetic analysis of the Italian HBOC pedigree. Fig. 1 shows the HBOC pedigree from Italy analyzed in this study. Patient 1 was born in 1931; at the age of 27 an undefined 'adenomatous nodule' was surgically removed from her left breast. At age 47 she was diagnosed with bilateral breast cancer; a right radical mastectomy and a left quadrantectomy were performed to remove a $\mathrm{T} 3 \mathrm{~N} 1 \mathrm{~b}+$ and a T1N0- lesion, respectively; surgery was followed by chemotherapy. Ten years later she was again hospitalized for removal of a left T1N0- lesion by radical mastectomy. On the same occasion, 
Table II. Mutations and polymorphisms detected in the BRCAl and BRCA2 genes in patient 1 from the Italian pedigree.

\section{BRCA1}

\begin{tabular}{|c|c|c|c|c|}
\hline Exon & $\begin{array}{l}\text { Nucleotide } \\
\text { change }\end{array}$ & $\begin{array}{c}\text { AA } \\
\text { change }\end{array}$ & $\begin{array}{l}\text { Sequence } \\
\text { variant }\end{array}$ & $\begin{array}{c}\text { Times reported in BIC database } \\
\text { or literature reference }\end{array}$ \\
\hline 5 & c. $190 \mathrm{~T}>\mathrm{C}$ & Cys64Arg & Mutation & 6 \\
\hline 8 & c. $442-34 \mathrm{C}>\mathrm{T}$ & & Intronic & 9 \\
\hline 11 & c. $2082 \mathrm{C}>\mathrm{T}$ & $\mathrm{p}=$ & Synonymous & 14 \\
\hline 11 & c. $2311 \mathrm{~T}>\mathrm{C}$ & $\mathrm{p}=$ & Synonymous & 25 \\
\hline 11 & c. $2612 \mathrm{C}>\mathrm{T}$ & p.Pro871Leu & Missense & 26 \\
\hline 11 & c. $3113 \mathrm{~A}>\mathrm{G}$ & p.Glu1038Gly & Missense & 37 \\
\hline 11 & c. $3548 \mathrm{~A}>\mathrm{G}$ & p.Lys1183Arg & Missense & 33 \\
\hline 13 & c. $4308 \mathrm{~T}>\mathrm{C}$ & $\mathrm{p}=$ & Synonymous & 35 \\
\hline 16 & c. $4956 \mathrm{G}>\mathrm{A}$ & p.Met1652Ile & Missense & 36 \\
\hline 17 & c. $5074+65 \mathrm{G}>\mathrm{A}$ & & Intronic & Song et al (ref. 22) \\
\hline
\end{tabular}

\section{BRCA2}

\begin{tabular}{rlllr}
\hline Exon & \multicolumn{1}{c}{$\begin{array}{c}\text { Nucleotide } \\
\text { change }\end{array}$} & $\begin{array}{c}\text { AA } \\
\text { change }\end{array}$ & $\begin{array}{c}\text { Sequence } \\
\text { variant }\end{array}$ & Times reported in BIC database \\
\hline 2 & c.-26G $>$ A & & Non-coding & 12 \\
11 & c.3396A $>$ G & $\mathrm{p}=$ & Synonymous & 8 \\
11 & c.3807T>C & $\mathrm{p}=$ & Synonymous & 3 \\
11 & c.6841+80_83delTTAA & & Intronic & 1 \\
\hline
\end{tabular}

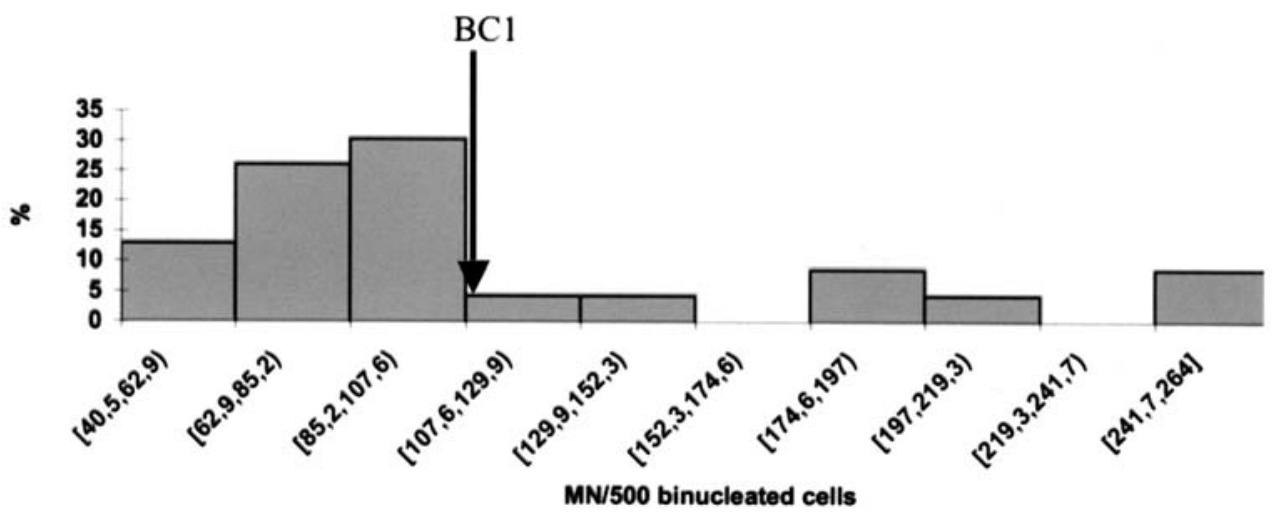

Figure 4. Frequency distribution of micronucleus formation (number of micronuclei/500 binucleated cells) in 23 EBV-immortalized control cell lines homozygous for the wild-type thymidine at position c.190 in the BRCA1 gene. The micronucleus score of the BC1 EBV-immortalized cell line (109 \pm 28 micronuclei/500 binucleated cells), homozygous for the c.190T>C mutation, is indicated with an arrow.

diffuse ovarian dysplastic lesions were reported. The patient underwent post-surgery standard chemotherapy treatment. The patient is in long-term remission since 1989. Patient 2 was diagnosed with breast dysplasia over 15 years ago, and is presently disease-free. Patient 3 was diagnosed with ovarian cancer (pT N G2) in 1988 at the age of 58, underwent surgical removal and chemotherapy, and is presently in remission. Patients 1, 2 and 3 are daughters of patient 4, who died of ovarian cancer at the age of 46. Patients 5 and 6, sister and mother of patient 4 , also died of ovarian cancer, both around the age of 60 .
All patients gave their informed consent for genetic analysis and publication of anonymous data. Patient 1 gave her consent for thorough $B R C A 1$ and $B R C A 2$ analysis. Genomic DNA was purified from peripheral blood lymphocytes and subjected to high-resolution melting analysis. Table II lists the mutations and polymorphisms identified in this patient. The c.190T>C mutation in exon 5 of BRCA1, generating the Cys64Arg amino acid substitution, was detected (Fig. 2). Moreover, nine BRCAI and four $B R C A 2$ known sequence variants, detected with high allele frequencies (19.57-38.26\%) in diverse European control populations (e.g., 23), were found. All sequence variants were 
cross-referenced with the Breast Cancer Information Core (BIC; http://research.nhgri.nih.gov/projects/bic/) (Table II), and all except two were classified as not clinically significant by BIC. c.6841+80_83delTTAA was the only variant not classified as non-significant; it is reported only once and classified as unknown. This is not unusual, since it is located 80 bp away from exon junction, and the variants commonly reported in BIC are up to $50 \mathrm{bp}$ away from exon junctions. This variant is present in a high percentage of healthy women with no family history of breast cancer [allele frequency in a central-European population: $38.26 \%$; (23)], thus it can be classified as non-significant.

Together with the missense Cys64Gly substitution, Cys64Arg is listed as being of unknown clinical significance in the BIC database. However, the Cys64Tyr substitution is defined as clinically important.

The functional relevance of the Cys64Arg substitution is described in the following section. Analysis of the polymorphisms detected in patient 1 , showed the presence of the c. 2612C $>$ T (Pro871Leu) missense polymorphism in BRCA1, and of the c.-26G $>$ A noncoding variant in BRCA2 (Table II). The significance of these mutations will be discussed in the following chapter.

\section{Structural and functional analysis of the c.190T $>C$ missense mutation}

Functional role of the Cys64Arg amino acid substitution. Cys61Gly and Cys64Gly are among the most prevalent BRCAl missense mutations, and are hypothesized to be responsible for hereditary breast cancer. These substitutions target specific metal-binding residues of BRCA1, and alter zinc coordination within the RING domain by presumably disrupting its three-dimensional coordination and in turn resulting into the impairment of BRCA1 interaction with its major functional ligand, BARD1 (reviewed in ref. 5).

The Cys61Gly mutation was shown to abolish metal binding to Site II of the RING domain of BRCA1, and disrupt BRCA1 homodimer formation (24). Moreover, the mutation resulted in increased proteolytic susceptibility of the carboxyterminal portion of the amino-terminal domain of BRCA1. The Cys61Gly mutation was also shown to abolish the in vitro ubiquitin-ligase activity displayed by the BRCA1-BARD1 complex (25).

The Cys64Gly mutation was demonstrated in a large African-American pedigree, characterized by early-onset breast/ovarian cancer, and subsequently in other AfricanAmerican families (1). Similarly to Cys64Gly and Cys61Gly, the Cys64Tyr mutation was shown to abolish the ubiquitin protein kinase activity of BRCA1, and to cause hypersensitivity to ionizing radiation (26). The same role can be hypothesized for the more rare Cys64Lys mutation (27)

So far, the Cys64Arg mutation has only been reported in Italy and in a single unrelated family in Poland $(15,28,29)$. Similarly to Cys61Gly, Cys64Gly and Cys64Tyr (26), the Cys64Arg substitution may cause disruption of BRCA1BARD1 interactions, thus affecting the ubiquitin-ligase activity of this heterodimeric complex.

The N-terminal region of BRCA1 shows the typical RING finger structure, belonging to the $\mathrm{C}_{3} \mathrm{HC}_{4} \mathrm{RING}$ finger domain family. In this family, two zinc ions are tetrahedrally coordinated by three cysteines and one histidine residue, and by four cysteine residues, respectively (see Fig. 3, upper panel, for a model). BRCA1 and BARD1 interact via an extensive anti-parallel 4-helix bundle interface formed by helices that flank the central RING motifs. From the analysis of the NMR structure of the BRCA1-BARD1 RING finger heterodimer (PDB ID: 1JM7), it appears that the Cys64 residue, belonging to the $\mathrm{C}_{3} \mathrm{H} \mathrm{Zn}$ coordination motif, is in close proximity to the Asp67 residue on the beta strand flanking the $\mathrm{Zn}$ environment. In the case of the Cys64Arg mutation, Arg64 is able to rotate towards Asp67, possibly forming a salt bridge (Fig. 3, lower panel). In turn, $\mathrm{Zn}$ is no longer stabilized by the tetrahedral coordination field formed by Cys39, His41, and Cys61. Alternatively, the $\mathrm{Zn}$ ion might form a complete coordination cage involving the $\operatorname{Arg} 64 \mathrm{~N}^{\varepsilon}$ atom. Interestingly, in the wildtype protein the beta strand opposite to the mutation (e.g., Cys39, Asp40, and His41) interacts with BARD1, with a hydrogen bond between Asp40 of BRCA1 and Asn98 of BARD1 (Fig. 3). It might therefore be suggested that the Cys64Arg mutation determines a rearrangement of the BRCA1 39-41 residues and destabilizes the BRCA1(Asp40)BARD1(Asn98) interaction (Fig. 3).

A similar hypothesis for the destabilization of the complex can be formulated for the Cys64Gly and Cys64Tyr mutants, described in other BRCA1 pedigrees (24-26). In the first case, the zinc atom should clearly dissociate from its coordination site. In the latter case, Tyr does not appear to be able to rotate towards the $\mathrm{Zn}$ ion, unless a dramatic rearrangement of the BRCA1 39-41 strand would take place.

BRCA1 involvement in DNA double-strand break repair. By likely disrupting the RING domain binding capacity, the Cys64Arg substitution might decrease the capacity of BRCA1 to stimulate DNA double-strand break repair in response to genotoxic agents such as ionizing radiation. This was shown for the Cys64Gly, Cys64Tyr and Cys61Gly substitutions (26). To test this hypothesis, we generated an EBV-immortalized lymphoblastoid cell line from peripheral blood lymphocytes of patient 1 from the Italian HBOC family, denominated BC1. Since BC1 cells showed an unexpected loss of heterozygosity during immortalization (Fig. 6, under investigation), they represented an optimal model for unbiased analysis of DNA DSB repair capacity. Cells were exposed to a dose of 2-Gy $\gamma$-rays, and subjected to the cytokinesis-block micronucleus $(\mathrm{MN})$ assay. Radiosensitivity of BC1 cells $(109 \pm 28$ micronuclei/500 binucleated cells) was compared to radiosensitivity of 23, randomly selected control EBV cell lines, T/T homozygous at locus 190 (codon 64). Fig. 4 shows the frequency distribution of MN scores of control EBV cell lines. Sixteen cell lines were more resistant to the genotoxic damage of ionizing radiation, whereas only 7 EBV control cell lines were found to be more radiosensitive. Thus, BC1 cells, carriers of the homozygous c.190T $>$ C mutation, were amongst the most radiosensitive cell lines investigated.

Effect of the c.190T>C mutation on BRCA1 mRNA splicing. The c.190T $>\mathrm{G}$ mutation was shown to generate aberrant splicing products of the BRCAl RNA (21). This occurred by two main mechanisms. First, the $\mathrm{T}>\mathrm{G}$ transversion activated a cryptic splicing donor site located within the coding region of exon 5, adjacent to the boundary with intron 5. Activation of the cryptic splicing donor site in the $\mathrm{T}>\mathrm{G}$ mutant results in 
$\mathbf{a}$

A

Natural splicing donor,

Exon 5, BRCA1

TTTCTTTATAATTTATAGATTTTGCATGCT GAAACTTCTCAACCAGAAGAAAGGGCCTTC ACAGTGTCCTTTATGTAAGAATGATATAAC CAAABgtatataatttggtaatgatgcta ggttggaagcaa

B

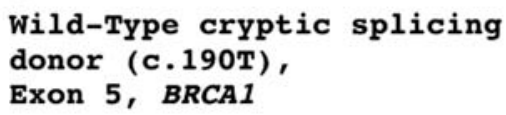

Wild-Type cryptic splicing donor (c.190T),

Exon 5, BRCA1

TTTCTTTATAATTTATAGATTTTGCATGCT GAAACTTCTCAACCAGAAGAAAGGGCCTTC ACAGTGTCCTTTA gtaagaatgatataac caaaaggtatataatttggtaatgatgcta ggttggaagcaa

C

c.190T>G mutant, activated cryptic splicing donor, Exon 5, BRCA1

TTTCTTTATAATTTATAGATTTTGCATGCT GAAACTTCTCAACCAGAAGAAAGGGCCTTC ACAGTGTCCTTTAGgtaagaatgatataac caaaaggtatataatttggtaatgatgcta ggttggaagcaa

D

c.190T>C mutant, putative cryptic splicing donor, Exon 5, BRCA1

TTTCTTTATAATTTATAGATTTTGCATGCT GAAACTTCTCAACCAGAAGAAAGGGCCTTC ACAGTGTCCTTTACgtaagaatgatataac caaaaggtatataatttggtaatgatgcta ggttggaagcaa

b

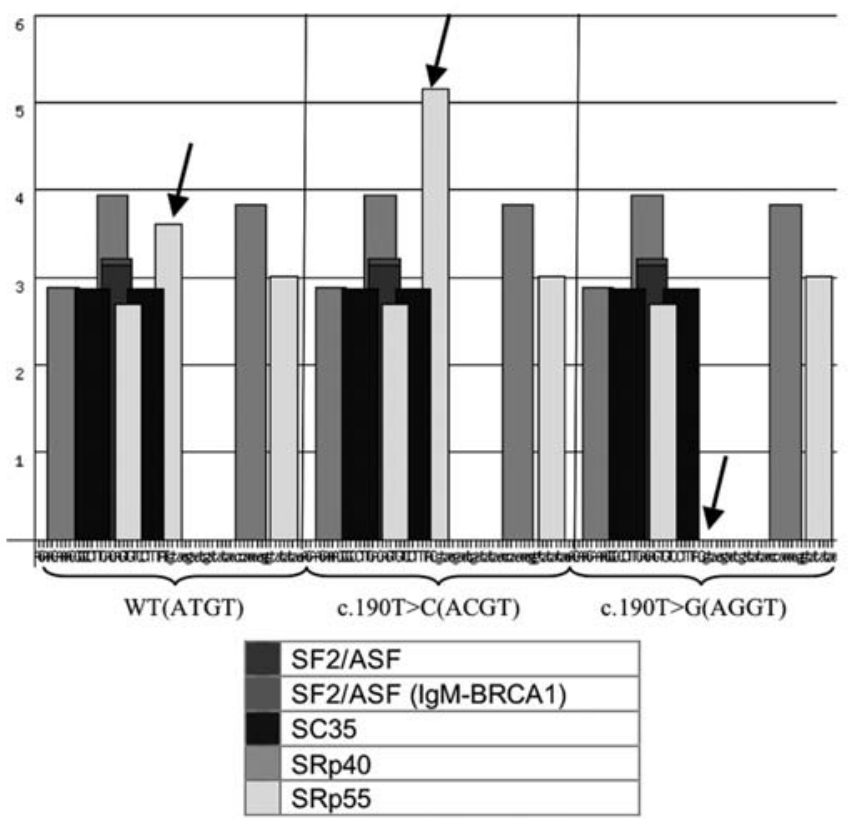

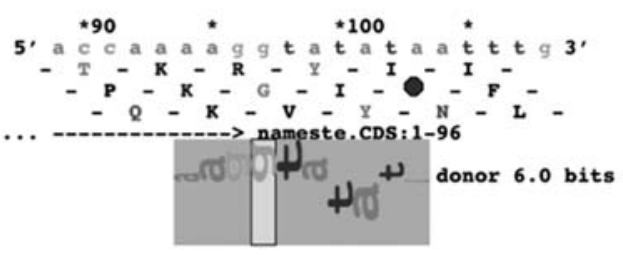
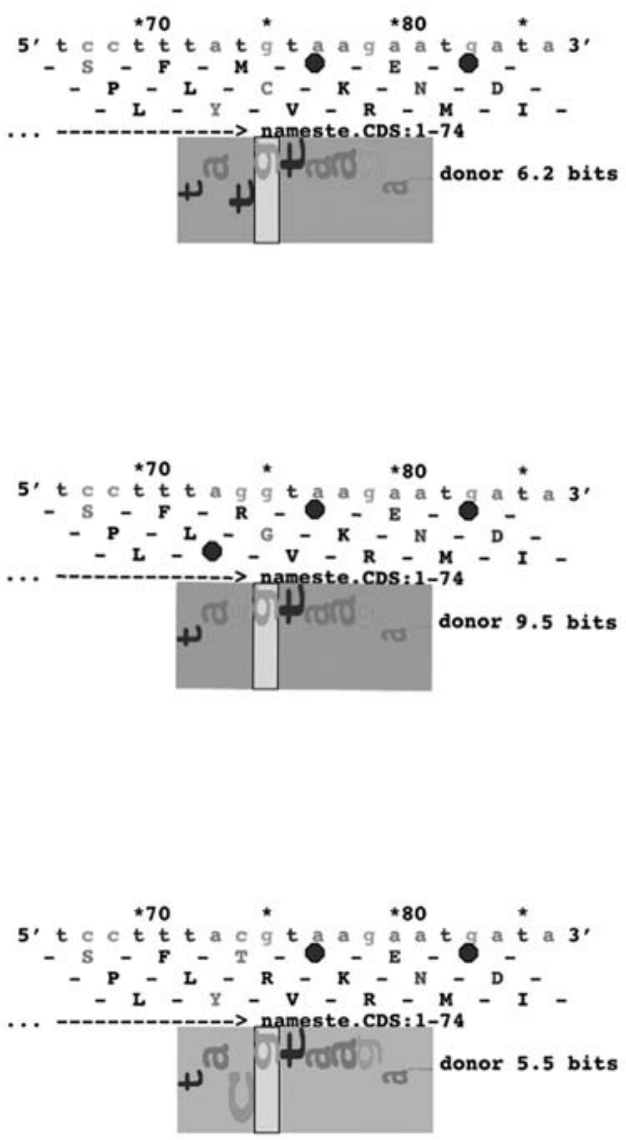

Figure 5. (a) Lister map, sequence walkers and information content of natural and cryptic splice donors of BRCAl exon 5. Exonic sequences are capitalized. (A) information content of the natural splicing donor (in dark grey). The position of the cryptic splicing donor is highlighted in light grey. (B) information content of the cryptic splicing donor (in light grey), in the absence of an activating mutation. The c.190T base is represented in dark grey. (C) cryptic splicing donor (in light grey) activated by the c.190T>G transversion (in dark grey). (D) putative cryptic splicing donor containing the c.190T>C mutation (in dark grey). The Schneider's lab tools (http://www. lecb.ncifcrf.gov/ toms/delila.html) were used to produce lister map and sequence walkers, and to calculate the information content of these splicing sites. (b) Effect of the c.190T $>\mathrm{G}$ and c.190T $>$ C mutations on high-score SRp55-binding motifs, in BRCA1 exon 5. SR motif analysis was performed using ESEfinder (http://rulai.cshl.edu/cgi-bin/tools/ESE3/esefinder.cgi? process=home). The c.190T $>\mathrm{G}$ mutation results in disruption of the SRp55 motif shown by the arrow. Conversely, the c.190T $>$ C mutation increases the score of the SRp55 motif from $\sim 3.6$ to $\sim 5.1$. SR motif types are listed in the legend. 


\section{$\begin{array}{lllllll}1 & 2 & 3 & 4 & 5 & 6 & 7\end{array}$}

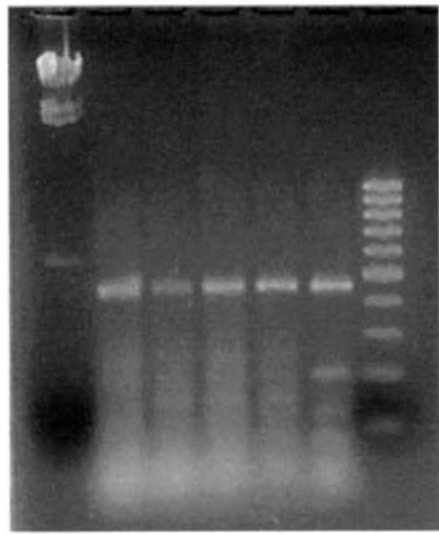

Figure 6. The c.190T $>\mathrm{C}$ missense mutation in BRCA1 exon 5 does not result in aberrant splicing, as shown by the presence of a 456-bp fragment, generated by RT-PCR with primers annealing with specific sequences in exons 5 (forward primer) and 6 [reverse primer; for primer sequences, see Materials and methods and (21)]. Lane 1: HindIII digest DNA marker; Lanes 2-4: EBV-immortalized, c.190T-homozygous cell lines from 3 control healthy individuals (selected randomly from the collection of 23 lines described in Materials and methods). Lanes 5 and 6: EBVimmortalized $\mathrm{BC} 1$ cell line from patient 1 (Italian pedigree). The small 200 bp fragment shown in lane 6 was sequenced, and represented a nonspecific amplicon. Lane 7: 100-bp marker. Template RNA was purified from BC1 cells, harboring the heterozygous c.190T $>C$ mutation. Correct junction of exon 5 and exon 6 extremities in a BRCA1 transcript from BC1 cells, confirmed by sequencing of the 456-bp fragment purified from this gel, is shown in the electropherogram.

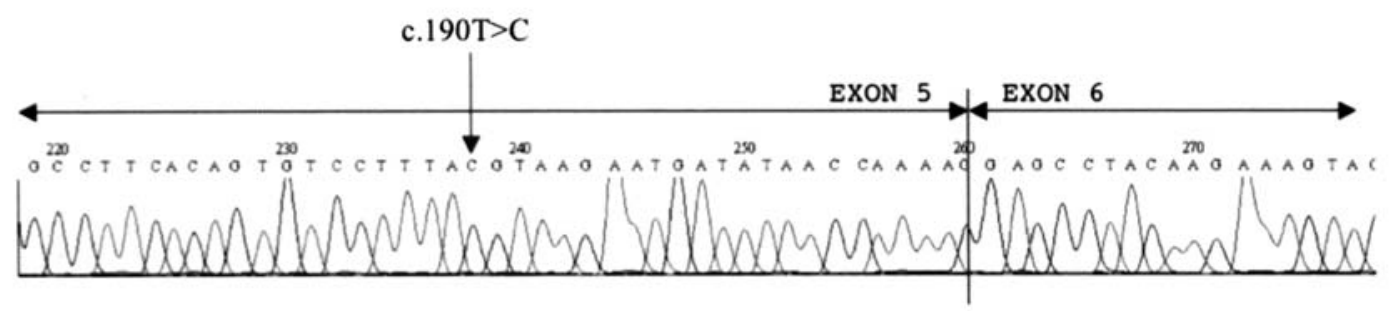

the deletion of 22 bases from exon 5, and in the generation of an aberrant, frame-shifted transcript, predicted to create an UGA premature termination codon at position 80 within exon 6 (21).

Surprisingly, in addition to the activation of a cryptic splicing site, the c.190T $>$ G mutation was shown to disrupt a high-score SRp55-binding exonic splicing enhancer (ESE) motif (position 189-193), likely involved in the normal splicing mechanism of intron 5 (21).

To investigate the influence of the $\mathrm{c} .190 \mathrm{~T}>\mathrm{C}$ mutation in $B R C A 1$ splicing, we compared by information analysis the information content (IC) of the natural BRCAl exon 5 splicing donor with the corresponding value of the putative splicing sites generated by the c.190T $>$ G and c.190T $>C$ mutations.

Fig. 5 shows that the natural splicing donor of $B R C A 1$ exon 5 is characterized by an information content (IC) of 6.0 bits. Interestingly, the wild-type cryptic donor (c.190T), located 22 bases upstream the exon 5-intron 5 boundary, shows a slightly higher information content ( 6.2 bits, Fig. 5a). The choice of the natural splicing donor over the cryptic one, is likely determined by the presence of splicing modulators, like the SRp55 motif described above, disrupted by the c. $190 \mathrm{~T}>\mathrm{G}$ mutation (21).

In the presence of the c.190T $>\mathrm{G}$ mutation, the score of the cryptic splice donor increases markedly, to a value of 9.5 bits. Since the likelihood of a splicing event has been shown to correlate with the amount of information contained in competing, alternative splice sites (30), our informationcontent analysis confirms the data described by Yang and coworkers (21), and shows that the c.190T $>$ G mutation, by increasing the IC of a cryptic splice donor, is responsible for the generation of alternatively-spliced, aberrant transcripts of the BRCAl gene.

In striking contrast with this evidence, information analysis showed that the $\mathrm{c} .190 \mathrm{~T}>\mathrm{C}$ mutation decreases the information content of the cryptic splicing donor from 6.2 to a value of 5.5 bits, thus diminishing the likelihood of aberrant splicing of the BRCAl RNA.

This hypothesis was strongly supported by analysis of the ESE motifs likely concurring to exon 5 splicing. Fig. 5b shows that, as shown by Yang et al (21), the c.190T>G mutation results in disruption of a high-score SRp55-binding ESE motif. However, the c.190T $>C$ mutation not only preserves the binding capacity of the ESE motif, but also further increases its score from $\sim 3.6$ to $\sim 5.1$.

In summary, whereas the c.190T $>\mathrm{G}$ mutation increases the information content of the competing, cryptic splice site located 22-nt upstream of the natural donor of exon 5, and simultaneously disrupts a high-score SRp55 binding site, working in normal conditions to the advantage of normal splicing of intron 5, the c.190T >C mutation preserves and even strengthens the natural splicing scenario of BRCAl intron 5, by decreasing the information content of the cryptic splice donor, and concurrently increasing the strength of the SRp55 ESE motif.

To confirm these in silico data, we analyzed by RT-PCR the splicing pattern and transcript sequence of the exon 5-exon 6 boundary, using total RNA purified from the $\mathrm{BC} 1$ mutant cell line. RT-PCR performed with the same primers, annealing with specific sequences in exon 5 and exon 6, used by Yang et al in their splicing study (21), yielded the expected fragment of $456 \mathrm{bp}$ (Fig. 6). The experiment was repeated 6 times with varying stringency conditions, but no fragments indicative of aberrant splicing were detected. Sequencing of the 456-bp PCR fragment amplified from BC1 cells confirmed a correct splicing pattern of BRCAl, at the level of exons 5 and 6 (Fig. 6).

Possible role of single nucleotide polymorphisms in BRCA1 and BRCA2, in the generation of the phenotype observed in the Italian HBOC family. In the literature, the typically Italian 


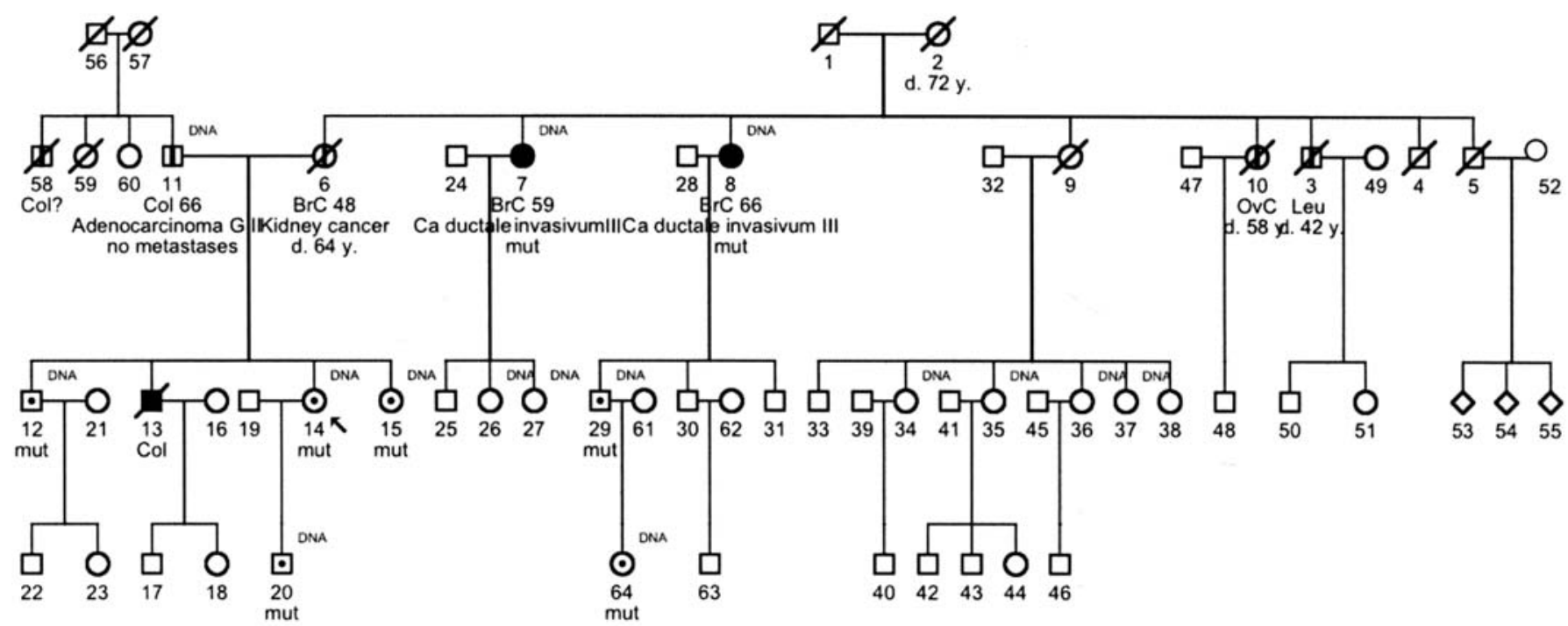

Figure 7. Pedigree of a Polish family characterized by prevalent breast neoplasms in carriers of the c.190T>C mutation. BrC, breast cancer; Leu, leukemia; Col, colon cancer; OvC, ovarian cancer; mut, mutation carrier.

Table III. Expression of significant polymorphisms in the $B R C A 1$ or $B R C A 2$ genes, in Italian and Polish pedigrees. ${ }^{a}$

Pedigree Pt.no. BRCA1, c.2612C >T BRCA2, c.-26G>A

\begin{tabular}{lccc}
\hline Italy & $\boldsymbol{1}$ & $\boldsymbol{C} / \boldsymbol{T}$ & $\boldsymbol{G} / \boldsymbol{A}$ \\
& 2 & $\mathrm{C} / \mathrm{C}$ & $\mathrm{G} / \mathrm{G}$ \\
\multirow{5}{*}{ Poland } & $\mathbf{3}$ & $\boldsymbol{C} / \boldsymbol{T}$ & $\boldsymbol{G} / \boldsymbol{A}$ \\
& $\boldsymbol{7}$ & $\boldsymbol{C} / \boldsymbol{T}$ & $\boldsymbol{G} / \boldsymbol{G}$ \\
& $\boldsymbol{8}$ & $\boldsymbol{T} / \boldsymbol{T}$ & $\boldsymbol{G} / \boldsymbol{G}$ \\
& 12 & $\mathrm{C} / \mathrm{T}$ & $\mathrm{A} / \mathrm{A}$ \\
& 14 & $\mathrm{C} / \mathrm{T}$ & $\mathrm{G} / \mathrm{G}$ \\
& 15 & $\mathrm{C} / \mathrm{T}$ & $\mathrm{G} / \mathrm{G}$ \\
& 20 & $\mathrm{C} / \mathrm{T}$ & $\mathrm{G} / \mathrm{A}$ \\
& 29 & $\mathrm{~T} / \mathrm{T}$ & $\mathrm{G} / \mathrm{G}$ \\
& 64 & $\mathrm{~T} / \mathrm{T}$ & $\mathrm{G} / \mathrm{A}$ \\
\hline
\end{tabular}

${ }^{\mathrm{a} C a n c e r}$ patients are emphasized with bold italic fonts.

c.190T>C mutation has to our best knowledge only been reported abroad in Poland, in a large family without Italian ancestry (15). This family is characterized by 3 cases of breast cancer (with two long-term survivors), one case of ovarian cancer (deceased) and one case of leukemia in a male proband (Fig. 7). For tentative comparison with a pedigree showing a potentially unrelated haplotype, we analyzed a total of 8 healthy or diseased probands from this Polish family.

As shown in a previous paragraph, analysis of the BRCAI/ $B R C A 2$ genotype of patient 1 from the Italian HBOC family (Table II), showed the presence of two heterozygous polymorphisms that were reported in the literature to be significantly linked to breast or ovarian cancer.

c.2612C $>T, B R C A 1$. The c. $2612 \mathrm{C}>\mathrm{T}$ missense variant in exon 11 of BRCA1, responsible for the Pro871Leu amino acid substitution, was shown to be the only polymorphic variant, out of a panel of 19 SNPs detected in BRCA1, whose frequency was significantly higher in patients $(42 \%)$ than in control individuals (28\%) (31). This was described in a population of 200 patients with breast/ovarian cancer screened for BRCAl mutations, drawn primarily from highrisk families and from isolated early-onset breast cancer cases. Comparison was made with a population of control individuals of diversified haplotypes.

Interestingly, the heterozygous c. $2612 \mathrm{C}>\mathrm{T}$ transition was detected in patients 1 and 3 from the Italian pedigree, but not in the cancer-free proband 2 (Table III), sister of these two patients. In breast cancer patients 7 and 8 from the Polish pedigree, the Pro871Leu substitution was found in a heterozygous and homozygous expression, respectively (Table III). All other healthy younger family members express this mutation in homo- or heterozygous forms. It would be of interest to extend these observations to larger patient populations and pedigrees, to ascertain the role of this polymorphism in the phenotypical expression of neoplasia (age of onset, intra-pedigree prevalence, penetrance and influence on prognosis).

c.-26G $>A, B R C A 2$. It has been demonstrated that the risk of ovarian cancer is higher for carriers of $B R C A 1$ mutations in the 5 ' portion of the gene, (e.g., Cys64Arg), although larger excesses of ovarian cancers were also observed near the 3 '-end of the gene (32). In Italian patients carrying the Cys64Arg mutation, the ratio between breast and ovarian lesions was shown to be $\sim 10$-fold (28). In contrast, the Italian HBOC pedigree analyzed in this study showed a high prevalence of ovarian cancers (80\%; Fig. 1). Moreover, in breast cancer patient 1 , ovarian precancerous lesions were detected. In families showing highly predominant ovarian lesions, the presence of modifier factors influencing the tumor localization may be hypothesized.

The c.-26G $>$ A transition within the 5'UTR of BRCA2 was initially identified as a polymorphism able to suppress the 
expression of the RNA transcribed from its carrier allele, without affecting transcription of the allele carrying the c.-26G variant (33). This allele-specific $B R C A 2$ suppression was demonstrated only in patients harboring a pathogenic mutation in $B R C A 2$, and not in individuals heterozygous for the c. $-26 \mathrm{G}>\mathrm{A}$ SNP, but lacking an additional deleterious mutation (33).

The c.-26G $>$ A polymorphism in BRCA2 was subsequently characterized as an ovarian cancer risk modifier in carriers of deleterious BRCAl mutations. In a study performed on 778 women carrying a BRCA1 germline mutation belonging to 403 families, a significant association of the c.-26A allele with increased risk of ovarian cancer was observed (Hazard ratio: $1.43,1.01-2.00,95 \% \mathrm{CI})(34)$. The authors postulated for the c. $-26 \mathrm{G}>\mathrm{A}$ variant of $B R C A 2$ a role as modifier of $B R C A 1$ associated ovarian cancer risk in $B R C A l$-mutation carriers, but not in the general population (34).

In the Italian pedigree shown in Fig. 1, characterized by predominant ovarian lesions, heterozygous expression of the c.-26G $>$ A polymorphism was documented in cancer patients 1 and 3 , showing bilateral breast cancer and diffuse ovarian dysplasia, and ovarian cancer, respectively (Table III). Cancerfree proband 2 , sister of patients 1 and 2 , shows a homozygous $\mathrm{G} / \mathrm{G}$ genotype. Interestingly, the patients of the Polish pedigree (Fig. 7), characterized by predominant breast cancers, showed a homozygous $\mathrm{G} / \mathrm{G}$ profile.

In summary, the c. $2612 \mathrm{C}>\mathrm{T}$ and the c. $-26 \mathrm{G}>\mathrm{A}$ common variants seem to segregate in the Italian and Polish pedigrees in a fashion coherent with their hypothetic role. However, the data shown in this study, analyzed in only two pedigrees, are only indicative of the potential association of the studied polymorphisms with disease penetrance and phenotypical modulation. It would be of interest to substantiate these preliminary observations with extensive studies performed on a large number of related and unrelated pedigrees.

In conclusion, the c.190T $>C$ (Cys64Arg) mutation analyzed in this study is presently described in the BIC database as a mutation of unknown clinical significance. Our study is indicative of a pathogenic role of this mutation, as shown in a preliminary survey of two unrelated familial breast and ovarian cancer pedigrees. In vitro and in silico functional experiments and observations indicate that the c.190T $>C$ mutation may induce profound modifications in the structure of the BRCA1 RING finger, but, unlike the Cys64Gly substitution, without affecting the normal splicing pattern of the $B R C A 1$ transcript. Additional polymorphic variants, shown in the literature to be associated with disease penetrance or ovarian cancer risk modulation, might have concurred to the peculiar phenotypic characteristics of the pedigrees examined in this preliminary study.

\section{Acknowledgements}

We are indebted to all patients described in this study, and their families and loved ones.

\section{References}

1. Castilla LH, Couch FJ, Erdos MR, et al: Mutations in the BRCA1 gene in families with early-onset breast and ovarian cancer. Nat Genet 8: 387-391, 1994.
2. Fackenthal JD and Olopade OI: Breast cancer risk associated with BRCA1 and BRCA2 in diverse populations. Nat Rev Cancer 7: 937-948, 2007.

3. Chen S and Parmigiani G: Meta-analysis of BRCA1 and BRCA2 penetrance. J Clin Oncol 25: 1329-1333, 2007.

4. Marroni F, Aretini P, D'Andrea E, et al: Penetrances of breast and ovarian cancer in a large series of families tested for BRCA1/2 mutations. Eur J Hum Genet 12: 899-906, 2004.

5. Baer R and Lee WH: Functional domains of the BRCA1 and BRCA2 proteins. J Mammary Gland Biol Neoplasia 3: 403-412, 1998.

6. Scully R, Chen J, Plug A, et al: Association of BRCA1 with Rad51 in mitotic and meiotic cells. Cell 88: 265-275, 1997.

7. Rosen EM, Fan S, Pestell RG and Goldberg ID: BRCA1 gene in breast cancer. J Cell Physiol 196: 19-41, 2003.

8. Chapman MS and Verma IM: Transcriptional activation by BRCA1. Nature 382: 678-679, 1996.

9. Wang H, Shao N, Ding QM, Cui J, Reddy ES and Rao VN: BRCA1 proteins are transported to the nucleus in the absence of serum and splice variants BRCA1a, BRCA1b are tyrosine phosphoproteins that associate with E2F, cyclins and cyclin dependent kinases. Oncogene 15: 143-157, 1997.

10. Wiwanitkit V: Molecular structure of BRCA1-estrogen receptor alpha-estrogen complex: relevance to breast cancer? Asian Pac J Cancer Prev 6: 561-562, 2005.

11. Irminger-Finger I and Jefford CE: Is there more to BARD1 than BRCA1? Nat Rev Cancer 6: 382-391, 2006.

12. Nishikawa H, Ooka S, Sato K, et al: Mass spectrometric and mutational analyses reveal Lys-6-linked polyubiquitin chains catalyzed by BRCA1-BARD1 ubiquitin ligase. J Biol Chem 279: 3916-3924, 2004

13. Agata S, Viel A, Della Puppa L, et al: Prevalence of BRCA1 genomic rearrangements in a large cohort of Italian breast and breast/ovarian cancer families without detectable BRCA1 and BRCA2 point mutations. Genes Chromosomes Cancer 45: 791-797, 2006.

14. Arnold N and Niederacher D: Denaturing high-performance liquid chromatography (DHPLC), an important mutation detection technique and mutation analysis of the breast and ovarian cancer predisposing genes BRCA1 and BRCA2. In: Genetic Variance Detection. Hecker KH (ed). DNA Press, PA, pp53-78, 2003.

15. Jakubowska A, Gorski B, Byrski T, et al: Detection of germline mutations in the BRCA1 gene by RNA-based sequencing. Hum Mutat 18: 149-156, 2001.

16. Brzovic PS, Rajagopal P, Hoyt DW, King MC and Klevit RE: Structure of a BRCA1-BARD1 heterodimeric RING-RING complex. Nat Struct Biol 8: 833-837, 2001.

17. Guex N and Peitsch MC: Swiss-model and the Swiss-PdbViewer: an environment for comparative protein modeling. Electrophoresis 18: 2714-2723, 1997.

18. Smith PJ, Zhang C, Wang J, Chew, SL, Zhang MQ and Krainer AR: An increased specificity score matrix for the prediction of SF2/ASF-specific exonic splicing enhancers. Hum Mol Genet 15: 2490-2508, 2006.

19. Cartegni L, Wang J, Zhu Z, Zhang MQ and Krainer AR: ESEfinder: a web resource to identify exonic splicing enhancers. Nucleic Acids Res 31: 3568-3571, 2003.

20. Baeyens A, Thierens H, Vandenbulcke K, De Ridder L and Vral A: The use of EBV-transformed cell lines of breast cancer patients to measure chromosomal radiosensitivity. Mutagenesis 19: 285-290, 2004.

21. Yang Y, Swaminathan S, Martin BK and Sharan SK: Aberrant splicing induced by missense mutations in BRCA1: clues from a humanized mouse model. Hum Mol Genet 12: 2121-2131, 2003.

22. Song CG, Hu Z, Wu J, et al: The prevalence of BRCA1 and BRCA2 mutations in eastern Chinese women with breast cancer. J Cancer Res Clin Oncol 132: 617-626, 2006.

23. Levacic Cvok M, Cretnik M, Musani V, Ozretic P and Levanat S: New sequence variants in BRCA1 and BRCA2 genes detected by high-resolution melting analysis in an elderly healthy female population in Croatia. Clin Chem Lab Med 46: 1376-1383, 2008.

24. Brzovic PS, Meza J, King MC and Klevit RE: The cancerpredisposing mutation $\mathrm{C} 61 \mathrm{G}$ disrupts homodimer formation in the NH2-terminal BRCA1 RING finger domain. J Biol Chem 273: 7795-7799, 1998.

25. Hashizume R, Fukuda M, Maeda I, et al: The RING heterodimer BRCA1-BARD1 is a ubiquitin ligase inactivated by a breast cancer-derived mutation. J Biol Chem 276: $14537-$ $14540,2001$. 
26. Ruffner H, Joazeiro CA, Hemmati D, Hunter T and Verma IM: Cancer-predisposing mutations within the RING domain of BRCA1: loss of ubiquitin protein ligase activity and protection from radiation hypersensitivity. Proc Natl Acad Sci USA 98: 5134-5139, 2001

27. Machackova E, Foretova L, Lukesova M, et al: Spectrum and characterisation of BRCA1 and BRCA2 deleterious mutations in high-risk Czech patients with breast and/or ovarian cancer. BMC Cancer 8: 140, 2008.

28. Aretini P, D'Andrea E, Pasini B, et al: Different expressivity of BRCA1 and BRCA2: analysis of 179 Italian pedigrees with identified mutation. Breast Cancer Res Treat 81: 71-79, 2003.

29. Carcangiu ML, Peissel B, Pasini B, Spatti G, Radice P and Manoukian S: Incidental carcinomas in prophylactic specimens in BRCA1 and BRCA2 germ-line mutation carriers, with emphasis on fallopian tube lesions: report of 6 cases and review of the literature. Am J Surg Pathol 30: 1222-1230, 2006.
30. Khan SG, Levy HL, Legerski R, et al: Xeroderma pigmentosum group $\mathrm{C}$ splice mutation associated with autism and hypoglycinemia. J Invest Dermatol 111: 791-796, 1998.

31. Durocher F, Shattuck-Eidens D, McClure M, et al: Comparison of BRCA1 polymorphisms, rare sequence variants and/or missense mutations in unaffected and breast/ovarian cancer populations. Hum Mol Genet 5: 835-842, 1996.

32. Gayther SA, Warren W, Mazoyer S, et al: Germline mutations of the BRCA1 gene in breast and ovarian cancer families provide evidence for a genotype-phenotype correlation. Nat Genet 11: 428-433, 1995.

33. Pyne MT, Brothman AR, Ward B, Pruss D, Hendrickson BC and Scholl T: The BRCA2 genetic variant IVS7 $+2 \mathrm{~T}>\mathrm{G}$ is a mutation. J Hum Genet 45: 351-357, 2000.

34. Hughes DJ, Ginolhac SM, Coupier I, et al: Common BRCA2 variants and modification of breast and ovarian cancer risk in BRCA1 mutation carriers. Cancer Epidemiol Biomarkers Prev 14: $265-267,2005$ 